\title{
Transmissões presentes em tratores agrícolas no Brasil
}

\author{
Transmissions present in agricultural tractors in Brazil
}

\section{Rodrigo Lampert Ribas ${ }^{*}$ José Fernando Schlosser $^{\text {II }}$ Ulisses Giacomini Frantz ${ }^{\text {III }}$ Marcelo Silveira de Farias $^{\mathrm{I}}$ Gustavo Heller Nietiedt ${ }^{\mathrm{II}}$}

\section{- NOTA -}

\section{RESUMO}

\begin{abstract}
O sistema de transmissão de um trator agrícola tem por principal finalidade transmitir a potência gerada no motor ao sistema hidráulico, à tomada de potência e às rodas motrizes. A caixa de câmbio é formada por uma série de engrenagens que permitem a correta seleção da velocidade $e$ do torque mais adequados a uma determinada operação. Assim, as transmissões podem ser classificadas, basicamente, em três categorias: mecânicas, hidrostáticas e hidrodinâmicas, sendo as transmissões mecânicas subdivididas em engrenagens deslizantes e sincronizadas. Este trabalho objetivou analisar os diferentes tipos de transmissões disponibilizados em 169 modelos de tratores fabricados no Brasil, com a finalidade de avaliar a real distribuição nas diferentes faixas de potência e estabelecer possíveis relações entre esses parâmetros. Como resultado, evidencia-se uma forte tendência de tratores agrícolas com potência menor que $111,8 \mathrm{~kW}$ possuírem transmissão do tipo mecânica, e tratores acima de $111,8 \mathrm{~kW}$, do tipo hidrostática.
\end{abstract}

Palavras-chave: caixa de câmbio, faixas de potência, classificação.

\begin{abstract}
The transmission system of an agricultural tractor has a main purpose to transmit the power generated in the hydraulic motor, the power take off and the drive wheels. The gearshift consists of a series of gears that allows the correct selection of speed and torque more appropriate to a particular operation. Then, the transmissions can be classified, basically, in three categories: mechanical, hydrostatic and hydrodynamic, being the mechanical transmissions subdivided in sliding gear
\end{abstract}

and synchronized. Then, the objective of this research was to analyze the different types of transmissions available in 169 models of tractors made in Brazil in order to evaluate the real distribution of them in different power ranges and establish possible correlations. As a result, it was shown a strong tendency for agricultural tractors with power less than $111.8 \mathrm{~kW}$ possessing mechanical transmission and tractors above $111.8 \mathrm{~kW}$ possessing the hydrostatic type.

Key words: gearshift, power range, classification.

Segundo a ASAE (1995), o trator agrícola é uma máquina de tração projetada e inicialmente recomendada para proporcionar potência aos implementos agrícolas. O trator agrícola é composto de motor, sistema de transmissão, sistema de direção e de sustentação e componentes complementares, onde são acoplados implementos e máquinas diversas (SCHLOSSER, 1997).

O sistema de transmissão é o conjunto de elementos que garantem a transmissão de potência do motor para os diferentes mecanismos a serem utilizados, como: o sistema hidráulico, a tomada de potência (TDP) e as rodas motrizes (MÁRQUEZ, 2004). A transmissão completa com a caixa de câmbio, o eixo traseiro, o acionamento da TDP, o acionamento do eixo dianteiro e os freios representa cerca de $30 \%$ do custo total do trator (ORTIZ-CAÑAVATE, 1994). Essa transmissão

${ }^{\mathrm{I} C u r s o}$ de Agronomia, Universidade Federal de Santa Maria (UFSM), 97105-900, Santa Maria, RS, Brasil. E-mail: rodrigoribasagronomia@yahoo.com.br. *Autor para correspondência.

"Departamento de Engenharia Rural, UFSM, Santa Maria, RS, Brasil.

IIIPrograma de Pós-graduação em Engenharia Agrícola (PPGEA), UFSM, Santa Maria, RS, Brasil. 
ocorre por meio do uso ou não da embreagem, a qual tem a função de conectar ou desconectar o motor à caixa de câmbio. A caixa de câmbio é formada por uma série de engrenagens que permitem a seleção da velocidade e do torque mais adequados para uma determinada operação, preconizando assim uma maior economia de combustível, bem como o aumento da eficiência operacional (SCHLOSSER, 1997). Assim, as transmissões podem ser divididas, basicamente, em três categorias: mecânicas, hidrostáticas e hidrodinâmicas.

A transmissão do tipo mecânica contém engrenagens que se deslocam em eixos com ranhuras para encaixarem-se umas às outras; logo, a velocidade de saída depende do número de pares engatados e do número de dentes das engrenagens. Nesse tipo de transmissão, a caixa de câmbio pode ser classificada, de acordo com o engrenamento, em: engrenagem deslizante e sincronizada. Na transmissão mecânica do tipo engrenagem deslizante, a marcha é selecionada antes do início do trabalho, segundo o critério do operador, sendo necessária a parada do trator para que se efetue a troca de marchas. Na transmissão mecânica do tipo sincronizada, qualquer marcha pode ser selecionada com o trator em movimento, pois anéis próprios acertam a velocidade de giro das engrenagens a serem acopladas. Para operações em que é exigida uma maior demanda de potência (aração, escarificação, etc), quando se deseja efetuar a troca de marchas durante a operação, torna-se necessário o acionamento da embreagem nos tratores com transmissões mecânicas, acarretando uma parada imediata do trator.

As configurações de transmissões hidrostáticas utilizadas em tratores agrícolas são baseadas em pacotes de discos com embreagens que direcionam o movimento da potência e do torque diferentemente dentro do sistema de transmissão, graças aos princípios de pressão hidrostática. Esse sistema torna possível a redução da velocidade e consequente ampliação do torque sem a necessidade de paradas do trator ou acionamentos da embreagem, o que aumenta a eficiência operacional e otimiza a operação.

A transmissão do tipo hidrodinâmica tem o princípio de transferência de potência por meio da energia cinética de um fluído hidráulico; no entanto, atualmente, esse tipo de transmissão não equipa nenhum trator agrícola.

A vantagem de um câmbio que possibilite um maior número de marchas, em que estas possam ser acionadas com o trator em movimento sem a necessidade de sua parada ou o acionamento da embreagem, é que torna possível a realização de trabalhos nas velocidades preconizadas para cada tipo de operação, proporcionando, assim, maior qualidade ao trabalho, aumento da eficiência operacional e economia de combustível. Como desvantagens, citamse o maior custo de aquisição de tratores equipados com estes sistemas, a maior possibilidade de erro na seleção da marcha e a necessidade de treinamento do operador.

Neste trabalho, foram verificados os tipos de transmissões disponibilizados em 12 fabricantes, totalizando 169 modelos de tratores fabricados no Brasil, com a finalidade de avaliar a real distribuição nas diferentes faixas de potência e estabelecer possíveis relações entre esses parâmetros.

Os tratores foram classificados quanto a sua faixa de potência em: menor ou igual a $37 \mathrm{~kW}$, entre $38 \mathrm{e}$ $74 \mathrm{~kW}$, entre 75 e $110 \mathrm{~kW}$, entre 111 e $147 \mathrm{~kW}$ e maior ou igual a $148 \mathrm{~kW}$, e as transmissões foram classificadas em três tipos: mecânicas (engrenagem deslizante e sincronizada) e hidrostáticas. Os dados foram adquiridos por meio de informações fornecidas pelos fabricantes de tratores no Brasil e posteriormente serviram de base para a elaboração de um banco de dados que tornou possível a comparação das faixas de potência com os tipos de transmissões.

Como resultado, a figura 1A mostra que, quanto à potência dos tratores, 65 dos 169 modelos analisados estão na faixa entre 38 e $74 \mathrm{~kW}, 36$ menor ou igual a $37 \mathrm{~kW}, 35$ entre 75 e 110kW, 24 entre 111 e 147kW e apenas nove maior ou igual a $148 \mathrm{~kW}$. Esses valores podem ser justificados devido ao mercado pretendido por cada empresa fabricante de tratores, uma vez que tais empresas trabalham com enfoques de mercado distintos no que se refere ao porte de seus tratores produzidos, estabelecendo nichos de mercado preferenciais. Ainda na figura 1A, dos 169 tratores analisados, o tipo de transmissão sincronizada é o que predomina nos tratores comercializados no Brasil, estando presente em 78 modelos.

De todas as faixas de potências comparadas, em nenhuma foi encontrado o tipo de transmissão hidrodinâmica. Na faixa de potência menor ou igual a $37 \mathrm{~kW}$, o tipo de transmissão engrenagem deslizante está presente em $83 \%$ dos tratores e apenas $17 \%$ são do tipo sincronizada, não estando presente a transmissão do tipo hidrostática. Na faixa entre 38 e $74 \mathrm{~kW}$, predomina o tipo de transmissão sincronizada, presente em $69 \%$ dos tratores, seguida da engrenagem deslizante, com $25 \%$, e da hidrostática, com $6 \%$. Entre 75 e $110 \mathrm{~kW}$, a transmissão sincronizada equipa $57 \%$ dos modelos de tratores; a hidrostática, $29 \%$, e a engrenagem deslizante, $14 \%$. Entre 111 e $147 \mathrm{~kW}$, a transmissão hidrostática aparece em 50\% dos modelos, seguidos da sincronizada, com $29 \%$, e da engrenagem 


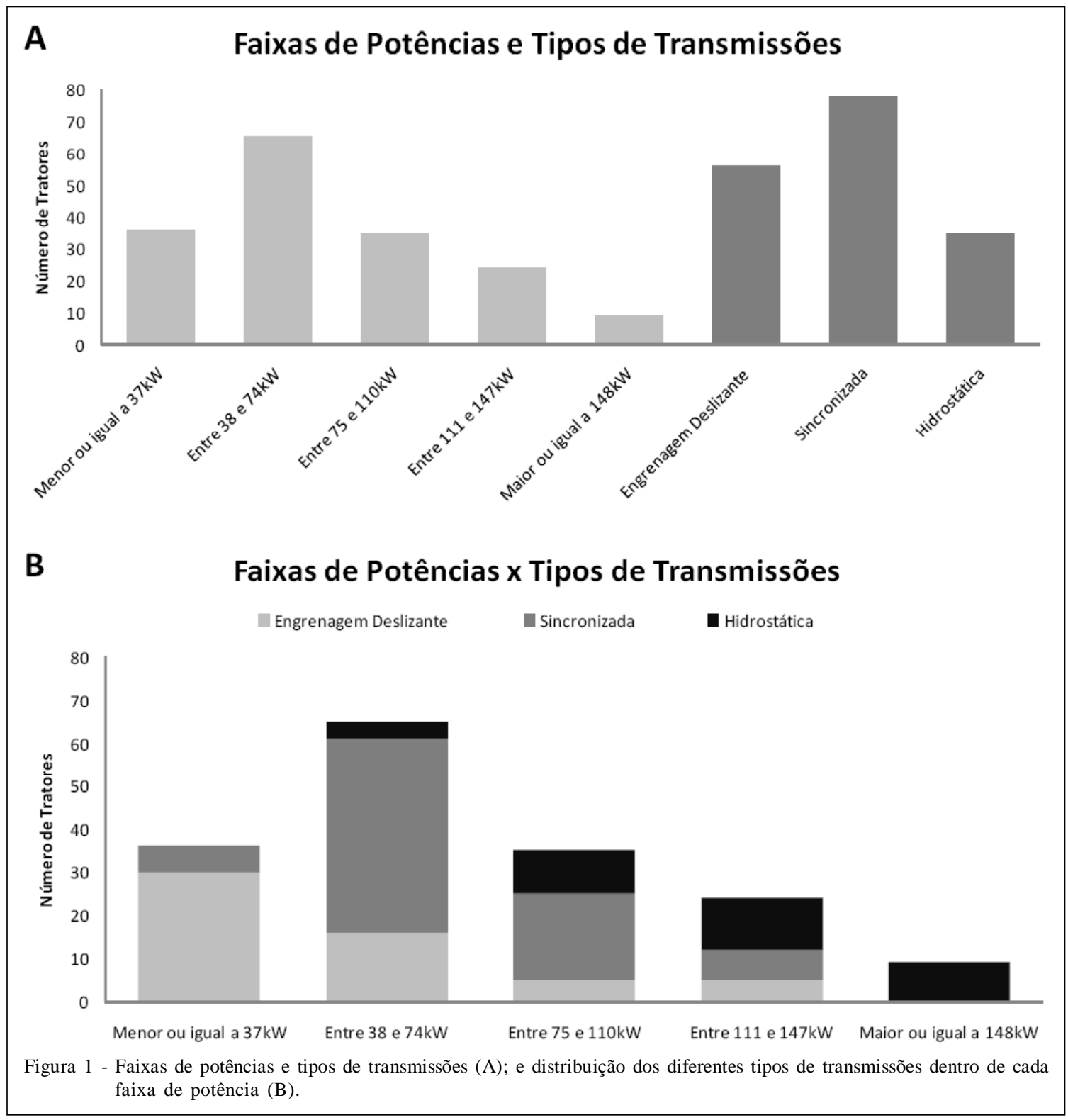

deslizante, com $21 \%$. Nos tratores com potência maior ou igual a $148 \mathrm{~kW}$, foi encontrado somente o tipo de transmissão hidrostática. Em linhas gerais, a transmissão do tipo sincronizada equipa $46 \%$ dos modelos de tratores; a engrenagem deslizante, $33 \%$; e a hidrostática, $21 \%$.

Esses resultados, expressos em percentuais, são apresentados em dados numéricos na figura $1 \mathrm{~B}$, evidenciando uma forte tendência de: tratores agrícolas com potência menor que $111,8 \mathrm{~kW}$ possuir transmissão do tipo mecânica (engrenagem deslizante e sincronizada) e tratores acima de $111,8 \mathrm{~kW}$, do tipo hidrostática. Tal situação deve-se ao fato de que, em faixas mais elevadas de potência, o custo da transmissão é diluído no preço total do trator, viabilizando a utilização desse recurso.

$\mathrm{Na}$ figura 2, estão representados os dados percentuais dos tratores em diferentes faixas de potência em relação aos tipos de transmissões. É possível constatar uma clara tendência, em que tratores de maior potência apresentam-se equipados com sistemas de transmissão mais modernos, como as transmissões hidrostáticas, figurando em $100 \%$ dos tratores situados na faixa de potência maior ou igual a $148 \mathrm{~kW}$. Da mesma forma, percebe-se que, em tratores de menor potência, há o predomínio de sistemas de 


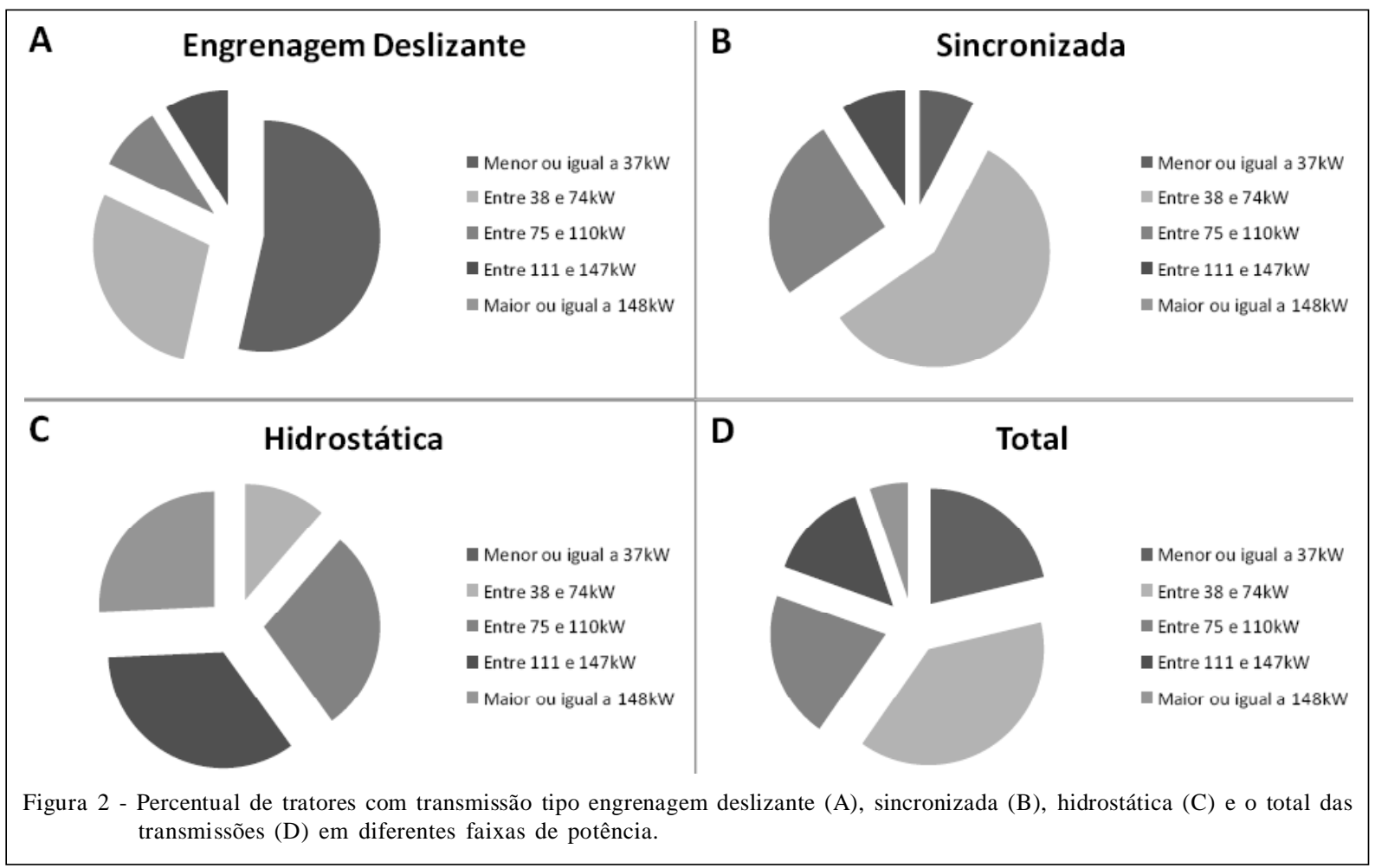

transmissão mais simples, como as transmissões por engrenagens deslizantes, figurando em cerca de $83 \%$ dos tratores situados na faixa de potência menor ou igual a $37 \mathrm{~kW}$. Finalmente, nota-se que os tratores pertencentes às faixas intermediárias de potência apresentam-se, em sua maioria, equipados com transmissões sincronizadas, em que cerca de 69, 57 e $29 \%$ dos tratores situados, respectivamente, nas faixas de potência de 38 a $74 \mathrm{~kW}, 75$ a $110 \mathrm{~kW}$ e 111 a $147 \mathrm{~kW}$, apresentam esse tipo de transmissão.

Os tratores agrícolas nacionais com potência menor que $111,8 \mathrm{~kW}$ possuem transmissão do tipo mecânica (engrenagem deslizante e sincronizada), e tratores acima de $111,8 \mathrm{~kW}$ possuem transmissão do tipo hidrostática.

Cada fabricante de tratores gera, em seus meios de informação, nomes específicos para suas transmissões, no entanto por vezes não consideram a classificação das transmissões apresentada anteriormente. Assim, salienta-se a necessidade de padronização da nomenclatura desses constituintes, a fim de melhor esclarecer as opções ofertadas ao consumidor.

\section{REFERÊNCIAS}

ASAE - American Society of Agricultural Engineer. Definitions of powered lawn and garden equipment. St. Joseph: ASAE, 1995. 826p. (ASAE Standard S323.2).

MÁRQUEZ, L. Las transmissiones: el escalonamiento de las marchas. Agrotécnica, Madrid, n.10, p.55-62, 2004.

ORTIZ-CAÑAVATE, J. Características técnicas de los tractores agrícolas. Vida Rural, Lisboa, n.6, p.32-34, 1994.

SCHLOSSER, J.F. Máquinas agrícolas. Santa Maria: Departamento de Engenharia Rural, 1997. 220p. (Caderno Técnico). 